\title{
COLLISIONS ARE OF FIRST CATEGORY ${ }^{1}$
}

\author{
DONALD G. SAARI
}

ABSTRACT. It is shown that the set of initial conditions leading to collisions in the $n$-body problem of Newtonian mechanics is of (Baire) first category.

1. Introduction. Motivated by a conjecture due to Littlewood [ 1, Problem 13], it was shown in some earlier papers [3], [4] that the set of initial conditions leading to collisions in the $n$-body problem of Newtonian mechanics is small in the sense of (Lebesgue) measure. Here we shall show that this set is small in a topological sense; namely, we shall show that this set is of (Baire) first category. The combination of the two results gives us the following statement.

Theorem 1. The set of initial conditions leading to collisions in the $n$-body problem has (Lebesgue) measure zero and is of (Baire) first category.

The result holds as stated whether physical space is a plane or a three dimensional space. It does not hold for the linear $n$-body problem; that is, where physical space is a straight line. (In this setting it is known that all initial conditions lead to collisions. However, the statement does hold in the linear $n$-body problem for the set of initial conditions leading to collisions where at least four particles collide at the same point in physical space.) Phase space can be considered as being either the full phase space $\left(\mathbf{R}^{6 n}\right.$ for the three-dimensional problem, $\mathbf{R}^{4 n}$ for the planar problem), or the reduced phase space which is obtained by fixing the center of mass of the system.

With the above choices for phase space and the dimension of physical space, we shall prove

Theorem 2. The set of initial conditions leading to collisions is of first category.

Received by the editors October 26, 1973.

AMS (MOS) subject classifications (1970). Primary 70F 10, 70F 15; Secondary 58F05, 34A25, 34C 35 .

Key words and phrases. Celestial mechanics, $N$-body problem, singularities.

1 This work was partially supported by N.S.F . grant GP 32116 . 
We now state some of the known results as lemmas.

Lemma 1 [3], [4]. The set of initial conditions leading to collisions has (Lebesgue) measure zero.

The combination of Theorem 2 and Lemma 1 yields Theorem 1.

The major difficulty in the proof of Theorem 2 is the possible existence of noncollision singularities, where we say that a solution has a noncollision singularity at time $t^{*}$ if at least one position vector does not approach a definite limit in an inertial coordinate system as $t \rightarrow t^{*}$.

Before we characterize the behavior of the two types of singularities, we introduce the following notation. Assume there is an inertial coordinate system. Let $m_{k}, \mathbf{r}_{k}$, and $\mathbf{v}_{k}$ denote, respectively, the mass, position vector, and velocity vector of the $k$ th mass. Let $2 I=\Sigma m_{k} \mathbf{r}_{k}^{2}$ and $2 T=\Sigma m_{k} \mathbf{v}_{k}^{2}$.

Lemma 2 [5]. If a solution of the $n$-body problem has a noncollision singularity at time $t^{*}$, then $I \rightarrow \infty$ and $\lim \sup \mid t^{*}-t^{2-\epsilon} T=\infty$ as $t \rightarrow t^{*}$. Here $\epsilon$ is any positive constant.

That is, this type of a singularity is characterized by motion becoming unbounded in physical space in finite time.

Lemma 3 [2]. If a solution of the $n$-body problem has a collision singularity at time $t^{*}$, then $I$ has a definite limit and $T \sim A /\left|t-t^{*}\right|^{2 / 3}$ as $t \rightarrow t^{*}$, where $A$ is some positive constant.

We are now prepared to prove Theorem 2.

2. Proof of Theorem 2. Assume that the conclusion of Theorem 2 is false; that is, if set $G$ is the set of initial conditions leading to collisions, then assume that set $G$ is of the second category. Define set $G_{m}$, for $m=0, \pm 1, \pm 2, \cdots$, to be the set of initial conditions with the properties that (i) their solutions suffer their first singularity in the time interval $[m, m+1]$, and (ii) this singularity is due to collisions. Clearly, $G=\bigcup G_{m}$.

Since set $G$ is assumed to be of second category and it is a denumerable union of the sets $G_{m}$, there exists some integer $m$, say $m=N$, such that set $G_{N}$ is of second category. The time reversibility of the system allows us to assume that integer $N$ is nonnegative.

Let $\left\{\eta_{j}\right\}$ be an increasing sequence of positive numbers such that $\eta_{j} \rightarrow \infty$ as $j \rightarrow \infty$. Define set $G^{\eta}$ to be those initial conditions $p$ such that (i) $p \in G_{N}$ and (ii) if the solution corresponding to $p$ has its first singular- 
ity at time $t^{*}$, then the solution has the property that $I(t) \leq \eta_{j}$ for $t \in\left[0, t^{*}\right)$. According to Lemma 3 , the continuity of $I(t)$ on the interval $\left[0, t^{*}\right)$, and the fact that $\eta_{j} \rightarrow \infty$ as $j \rightarrow \infty$, we have that if $p \in G_{N}$, then there exists a term $\eta_{j}$ such that $p \in G^{\eta_{j}}$. Thus, $G_{N}=\bigcup G^{\eta}$.

Since set $G_{N}$ is a set of second category, there is some constant $\eta_{j}$, say $\eta_{j}=\eta$, such that the set $G^{\eta}$ is of second category. Define set $G^{\circ}$ to be the interior of the closure of set $G^{\eta}$. By the fact that $G^{\eta}$ is of second category, we have that set $G^{\circ}$ is a nonempty open set. Therefore, set $G^{\circ}$ has positive (Lebesgue) measure. Consequently, we have from Lemma 1 that there exists $p \in G^{\circ}$ such that $p \notin G$.

There are only two possibilities for initial condition $p$. Either the solution corresponding to $p$ has no singularities in the time interval $[0, N+1]$, or the solution has a noncollision singularity at time $t^{*} \in(0, N+1]$. In the first case, we have from the continuity of solutions with respect to initial conditions, that for $\epsilon>0$, there exists an open ball of initial conditions centered about $p$ with the property that as long as the solutions exist in the time interval $[0, N+1]$, they differ from the one given by $p$ by no more than $\epsilon$. The solution given by $p$ is bounded in $[0, N+1]$. This forces the other solutions to be bounded as long as they do not encounter a singularity. However, from Lemmas 2 and 3, if they did encounter a singularity, then the solution would become unbounded ( $T$ is unbounded in either case). Consequently, continuity with respect to initial conditions gives us an open ball centered about $p$ with the property that the corresponding solutions exist in the time interval $[0, N+1]$. Clearly, this open ball is disjoint from sets $G_{N}$ and $G^{\eta}$; leading to the contradiction that $p \notin G^{\circ}$.

Assume that the solution corresponding to point $p$ suffers a noncollision singularity at time $t^{*} \in(0, N+1]$. According to Lemma 2 , there is some time $t, t \in\left(0, t^{*}\right)$, such that $I(t) \geq 3 \eta$. Since the solution corresponding to initial condition $p$ exists in the time interval $[0, t]$, we have from the continuity of solutions with respect to initial conditions, that for any $\epsilon>0$ there exists an open ball centered about point $p$ with the properties that (i) solutions corresponding to points in this ball exist in the time interval $[0, t]$, and (ii) for any value of time in this interval the solutions differ from the one given by $p$ by no more than $\epsilon$ units. In particular, there exists an open ball centered about $p$ such that the corresponding solutions have the property $I(t) \geq 2 \eta$. Clearly, this open ball is disjoint from set $G^{\eta}$, contradicting the statement that $p \in G^{\circ}$. Since we have contradicted the assumption that set $G$ is of second category, we have completed the proof of Theorem 2 . 
3. Set of all singularities. The set of initial conditions leading to a singularity at some time is a $F_{\sigma}$ set. To see this, let $S_{N}$ be the set of initial conditions leading to a singularity at some time in the interval $[-N, N]$, where $N$ is a positive integer. The above assertion is a consequence of the following statement.

Theorem 3. Set $S_{N}$ is a closed set.

Proof. Assume there exists $p \in \overline{S_{N}}$ such that $p \notin S_{N}$. Then the solution corresponding to $p$ exists on the time interval $[-N, N]$. But, by continuity of solutions with respect to initial conditions, there exists an open ball of initial conditions about $p$ such that the corresponding solutions exist on the same time interval. This contradicts the existence of point $p$.

\section{REFERENCES}

1. J. E. Littlewood, Some problems in real and complex analysis, Heath, Lexington, Mass., 1968. MR 39 \#5777.

2. H. Pollard and D. G. Saari, Singularities of the n-body problem. I, Arch. Rational Mech. Anal. 30 (1968), 263-269. MR 37 \#7118.

3. D. G. Saari, Improbability of collisions in Newtonian gravitational systems, Trans. Amer. Math. Soc. 162 (1971), 267-271; erratum, ibid. 168 (1972), 521. MR $45 \# 4714$.

4. - Improbability of collisions in Newtonian gravitational systems. II, Trans. Amer. Math. Soc. 181 (1973), 351-368.

5. - Singularities and collisions of Newtonian gravitational systems. Arch. Rational Mech. Anal. 49 (1973), 311-320.

DEPARTMENT OF MATHEMATICS, NORTHWESTERN UNIVERSITY, EVANSTON, ILLINOIS 60201 